\title{
Optimal diffusion rate of species in flowing habitat
}

\author{
Benlong Xu* (D) and Nannan Liu
}

${ }^{*}$ Correspondence: bxu@shnu.edu.cn

Department of Mathematics, Shanghai Normal University,

Shanghai, 200234, P.R. China

\section{照 Springer}

\begin{abstract}
It is widely accepted that diffusive dispersal can permit persistence in an advective environment. This paper studies in some sense the optimal diffusion rate of species in a flowing habitat with hostile downstream boundary conditions. Firstly, we study the dependence of the critical length of the habitat on the dispersal rate $d$. It is shown that the critical length first decreases and then increases and asymptotically tends to infinity. Then there is a unique optimal diffusion rate $d_{0}$ for a single species to evolve. Then, by using this observation, we study the competition system of two species which are the same but only with different dispersal rates. We get an open finite interval, which is a neighborhood of $d_{0}$, such that, if one of the dispersal rates lies within the interval but the other rate falls outside, then competition exclusion occurs. If the two dispersal rates both lie within the interval, the one with an intermediate dispersal rate can always invade the other with its dispersal rate near the ends of the interval.
\end{abstract}

MSC: $35 \mathrm{~K} 57 ; 92 \mathrm{D} 25$

Keywords: flowing habitat; diffusion rate; globally asymptotically stable

\section{Introduction}

There are many species residing in flowing habitats, such as streams and rivers. As a result, the unidirectional water movement constantly drives the species out of the habitat. Why and how can populations persist in streams when they are constantly washed out downstream? This question, known as the 'drift paradox' in the literature, has received considerable attention [1-3]. Speirs and Gurney [4] argued that diffusive movement of the species can allow persistence in a flowing habitat by considering the following mathematical model:

$$
u_{t}=d u_{x x}-q u_{x}+u(r-u), \quad 0<x<L, t>0,
$$

with boundary conditions

$$
d u_{x}(0, t)-q u(0, t)=0, \quad u(L, t)=0, \quad t>0,
$$

where $u(x, t)$ denotes the population density at location $x$ and time $t, d$ is the diffusion rate, $L$ is the size of the habitat, $q$ is the effective speed of the current, and $r$ is the intrinsic

(c) The Author(s) 2017. This article is distributed under the terms of the Creative Commons Attribution 4.0 International License (http://creativecommons.org/licenses/by/4.0/), which permits unrestricted use, distribution, and reproduction in any medium, provided you give appropriate credit to the original author(s) and the source, provide a link to the Creative Commons license, and indicate if changes were made. 
growth of the species. For the boundary conditions, at the upstream end $x=0$, the species is assumed to satisfy the no-flux boundary condition, which means that no individual will pass through this boundary, while at the downstream end $x=L$, the species satisfy hostile boundary conditions, such as in the scenario stream to ocean.

Speirs and Gurney [4] obtained a critical length $L^{*}$ of the habitat and conclude that the species permits persistence if and only if $q<\sqrt{4 d r}$ and $L>L^{*}$. So the species can persist if and only if the diffusion rate $d$ is bigger than $\frac{q^{2}}{4 r}$, and the length of the habitat is bigger than the critical length $L^{*}$.

A natural question arises: is there an optimal diffusion rate $d$, in the sense that species adopting this diffusion rate will need the smallest critical length $L^{*}$ to persist?

To further explore the role of the diffusion rate in flowing habitats, Lou and Lutscher [5] suggested the following model of two competing species that are identical except for their diffusion rate:

$$
\begin{array}{ll}
u_{t}=d_{1} u_{x x}-q u_{x}+u(r-u-v), & 0<x<L, t>0, \\
v_{t}=d_{2} v_{x x}-q v_{x}+v(r-v-u), & 0<x<L, t>0 .
\end{array}
$$

The upstream end always imposes a no-flux boundary condition, that is,

$$
d_{1} u_{x}(0, t)-q u(0, t)=0, \quad d_{2} v_{x}(0, t)-q v(0, t)=0, \quad t>0
$$

For the downstream end, Lou and Lutscher [5] proposed three types of boundary conditions:

(I) no-flux (closed) boundary condition:

$$
d_{1} u_{x}(L, t)-q u(L, t)=0, \quad d_{2} v_{x}(L, t)-q v(L, t)=0, \quad t>0,
$$

(II) free flow (open) boundary condition:

$$
u_{x}(L, t)=0, \quad v_{x}(L, t)=0, \quad t>0,
$$

(III) hostile (lethal) boundary condition:

$$
u(L, t)=0, \quad v(L, t)=0, \quad t>0 .
$$

It is an interesting question how the diffusion rates $d_{1}$ and $d_{2}$ determine the dynamic behavior of system (1.3). For the free flow downstream condition (II), it is proved in [5] that the species with a higher diffusion rate will always prevail, that is, faster diffusion wins. Later on, Lou and Zhou [6] extended this result to a wider class of boundary conditions. Recently, Lam et al. [6] studied the system with no-flux downstream conditions (I), and showed that, when $r$ is a positive constant, the higher diffusion will always prevail, just as in the case of free flow downstream conditions. The reason for these results is that a sufficient intensity of random diffusion can better balance the overmatching of resources or even a net loss of individuals at the boundary.

It is natural to ask about the role of random dispersal in the situation of hostile downstream boundary conditions (III). It turns out that this situation is more complicated. On 
the one hand, the random dispersal balances the overmatching of resources and loss of individuals caused by drifting, while on the other hand, too strong dispersal also likely drives more individuals to the hostile end. Lou and Lustscher [5] conjectured that an intermediate diffusion rate seems most beneficial.

This paper is motivated by the question mentioned above. The rest of the paper is organized as follows. In Section 2, we state and prove our main results. Firstly, we explore the optimal diffusion rate for a single species to persist. We give a rigorous analytical proof that there exists a unique optimal diffusion rate, in the sense that a species adopting this diffusion rate will need the smallest length of critical habitat for persistence. Then we study the global dynamics of a two-species competition model. By using the optimal results for the single species, we get an optimal interval such that the species with its diffusion rate in this interval always prevails against its competitor with its diffusion rate beyond the interval. In Section 3, some concluding remarks and the discussion are given.

\section{Main results}

We consider firstly the following model:

$$
\left\{\begin{array}{l}
u_{t}=d_{1} u_{x x}-q u_{x}+u(r-u), \quad 0<x<L, t>0 \\
d u_{x}(0, t)-q u(0, t)=0, \quad t>0 \\
u(L, t)=0, \quad t>0, \\
u(x, 0)=u_{0}(x) \geq \not \equiv 0, \quad 0<x<L
\end{array}\right.
$$

where $u(x, t)$ denotes the population density at location $x$ and time $t, d$ is the diffusion rate, $L$ is the size of the habitat, $q$ is the effective speed of the water, and $r$ is the intrinsic growth rate of the species. We assume that $d, r, q, L$ are all positive constants.

Speirs and Gurney [4] obtained the critical length of the habitat by

$$
L^{*}=2 d \frac{\pi-\arctan \frac{\sqrt{4 d r-q^{2}}}{q}}{\sqrt{4 d r-q^{2}}},
$$

and conclude that the species permits persistence if and only if

$$
d>\frac{q^{2}}{4 r} \text { and } L>L^{*}
$$

We will explore the dependence of $L^{*}$ on the diffusion rate $d$. Let us first rewrite $L^{*}$ as a function of the diffusion rate $d$. We have

$$
L^{*}(d)=2 d \frac{\pi-\arctan \frac{\sqrt{4 d r-q^{2}}}{q}}{\sqrt{4 d r-q^{2}}}, \quad d>\frac{q^{2}}{4 r} .
$$

Some numerical simulations suggest that $L^{*}$ as the function of $d$ has a minimal point (see Figure 1). We will give a rigorous analytical proof of this fact.

Theorem 2.1 $L^{*}(d)$ has a unique critical point $d_{0}$ in $\left(\frac{q^{2}}{4 r},+\infty\right)$, such that $L^{*}(d)$ is strictly decreasing in $\left(\frac{q^{2}}{4 r}, d_{0}\right)$ and strictly increasing in $\left(d_{0},+\infty\right)$. Moreover, $\lim _{d \rightarrow \frac{q^{2}}{4 r}+0} L^{*}(d)=+\infty$, $\lim _{d \rightarrow+\infty} L^{*}(d)=+\infty$. 
Figure 1 The critical length $L^{*}$ of the habitat.

Parameters are $q=5, r=3$.

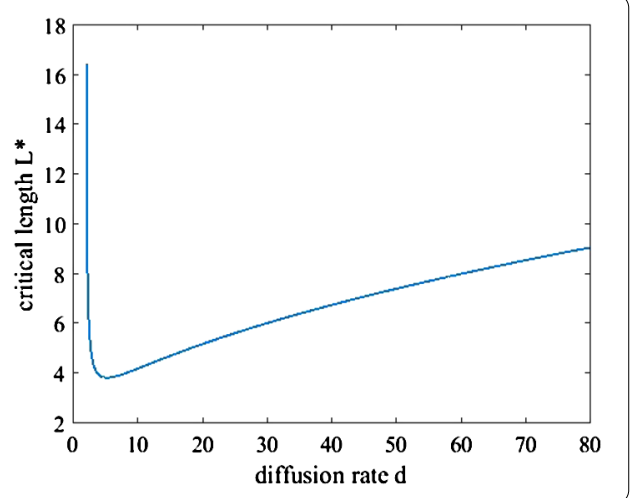

Proof We will discuss the dependence of $L^{*}$ on $d$ by the first and the second derivation. We have

$$
\begin{aligned}
L^{*^{\prime}}(d) & =\frac{\left(\pi-\arctan \frac{\sqrt{4 d r-q^{2}}}{q}\right)\left(4 d r-q^{2}\right)-q \sqrt{4 d r-q^{2}}}{\left(4 d r-q^{2}\right) \sqrt{4 d r-q^{2}}} \\
& \triangleq \frac{1}{\left(4 d r-q^{2}\right) \sqrt{4 d r-q^{2}}} f(d),
\end{aligned}
$$

where

$$
f(d)=\left(\pi-\arctan \frac{\sqrt{4 d r-q^{2}}}{q}\right)\left(4 d r-q^{2}\right)-q \sqrt{4 d r-q^{2}}
$$

A direct computation yields

$$
f^{\prime}(d)=4 r\left(\pi-\arctan \frac{\sqrt{4 d r-q^{2}}}{q}\right)-\frac{q}{d} \sqrt{4 d r-q^{2}}
$$

and

$$
f^{\prime \prime}(d)=-\frac{\left(4 d r-q^{2}\right) q^{3}}{d^{2}\left(4 d r-q^{2}\right) \sqrt{4 d r-q^{2}}}
$$

It is obvious from $(2.7)$ that $f^{\prime \prime}(d)<0$ for any $d>\frac{q^{2}}{4 r}$, and $f^{\prime}(d)$ is strictly decreasing for $d>\frac{q^{2}}{4 r}$. By (2.6), $\lim _{d \rightarrow+\infty} f^{\prime}(d)=4 r\left(\pi-\frac{\pi}{2}\right)=2 r \pi>0$, and $\lim _{d \rightarrow \frac{q^{2}}{4 r}+0} f^{\prime}(d)=4 r \pi>0$. It follows that $f^{\prime}(d)>0$ for any $d>\frac{q^{2}}{4 r}$, and thus $f(d)$ is strictly increasing for $d>\frac{q^{2}}{4 r}$. Since $\lim _{d \rightarrow \frac{q^{2}}{4 r}+0} f(d)=-q^{2} \pi<0$, and $\lim _{d \rightarrow+\infty} f(d)=+\infty$ by (2.5), there exists a unique number $d_{0}>\frac{q^{2}}{4 r}$, such that $f(d)<0$ if $d \in\left(\frac{q^{2}}{4 r}, d_{0}\right)$, and $f(d)>0$ if $d \in\left(d_{0},+\infty\right)$. It follows from (2.4) that $L^{*^{\prime}}(d)<0$ for $d \in\left(\frac{q^{2}}{4 r}, d_{0}\right)$, and $L^{*^{\prime}}(d)>0$ for $d \in\left(d_{0},+\infty\right)$. Thus $L^{*}(d)$ is strictly decreasing in $\left(\frac{q^{2}}{4 r}, d_{0}\right)$ and strictly increasing in $\left(d_{0},+\infty\right)$, and $d_{0}$ is its unique critical point.

Finally, the assertions $\lim _{d \rightarrow \frac{q^{2}}{4 r}+0} L^{*}(d)=+\infty$ and $\lim _{d \rightarrow+\infty} L^{*}(d)=+\infty$ can be verified directly by equation (2.3). This completes the proof.

Remark 2.1 As is well known, a species with diffusion rate $d>q^{2} /(4 r)$ can persist if and only if the habitat length $L$ is bigger than the critical length $L^{*}(d)$. Theorem 2.1 tells us that, 
for fixed $r$ and $q$, there exists an optimal diffusion rate $d_{0}$ which has a minimal critical length. A species adopting diffusion rate $d_{0}$ can persist with the smallest length of the habitat.

In the following, we focus our attention on the study of the two-species competition system. It turns out that Theorem 2.1 provides a tool to study the role of the diffusion rate in the competition system. Following [5], we consider the competition system

$$
\left\{\begin{array}{l}
u_{t}=d_{1} u_{x x}-q u_{x}+u(r-u-v), \quad 0<x<L, t>0, \\
v_{t}=d_{2} v_{x x}-q v_{x}+v(r-u-v), \quad 0<x<L, t>0, \\
d_{1} u_{x}(0, t)-q u(0, t)=0, \quad u(L, t)=0, \quad t>0, \\
d_{2} v_{x}(0, t)-q v(0, t)=0, \quad v(L, t)=0, \quad t>0, \\
u(x, 0)=u_{0}(x) \geq \not \equiv 0, \quad v(x, 0)=v_{0}(x) \geq \not \equiv 0, \quad 0<x<L .
\end{array}\right.
$$

The dynamic behavior of system (2.8) depends heavily on the existence and stability of the non-negative solutions of the corresponding steady state equation:

$$
\left\{\begin{array}{l}
d_{1} u_{x x}-q u_{x}+u(r-u-v)=0, \quad 0<x<L, \\
d_{2} v_{x x}-q v_{x}+v(r-u-v)=0, \quad 0<x<L, \\
d_{1} u_{x}(0)-q u(0)=0, \quad u(L)=0, \\
d_{2} v_{x}(0)-q v(0)=0, \quad v(L)=0 .
\end{array}\right.
$$

Steady state system (2.9) always has the trivial solution $(0,0)$. By [4] (also see [7] Lemma 2.2), steady state system (2.9) has a semi-trivial solution $(\tilde{u}, 0)$ if and only if $d_{1}>\frac{q^{2}}{4 r}$ and $L>L^{*}\left(d_{1}\right)$, where $\tilde{u}$ is the unique positive solution of the problem

$$
\left\{\begin{array}{l}
d_{1} u_{x x}-q u_{x}+u(r-u)=0, \quad 0<x<L, \\
d_{1} u_{x}(0)-q u(0)=0, \quad u(L)=0 .
\end{array}\right.
$$

Similarly, (2.9) has a semi-trivial solution (0, $\tilde{v})$ if and only if $d_{2}>\frac{q^{2}}{4 r}$ and $L>L^{*}\left(d_{2}\right)$.

By Theorem 2.1, $L^{*}(d)$ has a minimum critical point $d_{0}$, therefore we discuss the dynamics of (2.8) by two parts: $d_{0} \in\left(\frac{q^{2}}{4 r}, d_{0}\right)$ and $d \in\left(d_{0},+\infty\right)$.

Theorem 2.2 Assume that $r>0, q>0$, and $L>L_{0}$, where $L_{0}=L^{*}\left(d_{0}\right)=\min \left\{L^{*}(d): d>\frac{q^{2}}{4 r}\right\}$. Then there exist two constants $\underline{d}$, and $\bar{d}$ with $\frac{q^{2}}{4 r}<\underline{d}<d_{0}<\bar{d}<+\infty$, such that the following conclusions hold:

(a) if $d_{1} \in(\underline{d}, \bar{d})$, the semi-trivial steady state solution $(\tilde{u}, 0)$ exists. Furthermore, if $d_{2}$ is not in the interval $(\underline{d}, \bar{d}),(\tilde{u}, 0)$ is globally asymptotically stable for system $(2.8)$,

(b) if $d_{2} \in(\underline{d}, \bar{d})$, the semi-trivial steady state solution $(0, \tilde{v})$ exists. Furthermore, if $d_{1}$ is not in the interval $(\underline{d}, \bar{d}),(0, \tilde{v})$ is globally asymptotically stable for system (2.8),

(c) if $d_{1}$ and $d_{2}$ are not in the interval $(\underline{d}, \bar{d})$, then $(0,0)$ is globally asymptotically stable for system (2.8).

Proof By Theorem 2.1, $L^{*}(d)$ is strictly decreasing in $\left(\frac{q^{2}}{4 r}, d_{0}\right)$, strictly increasing in $\left(d_{0},+\infty\right)$, and $\lim _{d \rightarrow \frac{q^{2}}{4 r}+0} L^{*}(d)=+\infty, \lim _{d \rightarrow+\infty} L^{*}(d)=+\infty$. Since $L>L_{0}=L^{*}\left(d_{0}\right)$, there 
exist two constants $\underline{d}$, and $\bar{d}$ with $\frac{q^{2}}{4 r}<\underline{d}<d_{0}<\bar{d}<+\infty$, such that $L^{*}(\underline{d})=L^{*}(\bar{d})=L$. It follows that $L>L^{*}(d)$ if $d \in(\underline{d}, \bar{d})$, and $L \leq L^{*}(d)$ if $\frac{q^{2}}{4 r}<d<\underline{d}$, or $d \geq \bar{d}$.

(a) As is well known, positive solution $\tilde{u}$ of steady state equation (2.10) exists if and only if $d_{1}>\frac{q^{2}}{4 r}$ and $L>L^{*}\left(d_{1}\right)$, and this condition is actually satisfied if $d_{1} \in(\underline{d}, \bar{d})$. This establishes the existence of the semi-trivial steady state solution $(\tilde{u}, 0)$.

Next we show that, given any non-negative and not identical to zero initial data, $\left(u_{0}(x), v_{0}(x)\right),(u(x, t), v(x, t)) \rightarrow(\tilde{u}, 0)$ as $t \rightarrow+\infty$. By the maximum principle, $u(x, t)>0$ and $v(x, t)>0$ for any $x \in[0, L)$ and $t>0$. Therefore, by $(2.8), v(x, t)$ satisfies

$$
\left\{\begin{array}{l}
v_{t}<d_{2} v_{x x}-q v_{x}+v(r-v), \quad 0<x<L, t>0, \\
d_{2} v_{x}(0, t)-q v(0, t)=0, \quad t>0, \\
v(L, t)=0, \quad t>0, \\
v(x, 0)=v_{0}(x) \geq \not \equiv 0, \quad 0<x<L .
\end{array}\right.
$$

That is, $v(x, t)$ is a sub-solution of the equation

$$
\left\{\begin{array}{l}
Z_{t}=d_{2} Z_{x x}-q Z_{x}+Z(r-Z), \quad 0<x<L, t>0 \\
d_{2} Z_{x}(0, t)-q Z(0, t)=0, \quad t>0 \\
Z(L, t)=0, \quad t>0 \\
Z(x, 0)=v_{0}(x) \geq \neq \equiv 0, \quad 0<x<L .
\end{array}\right.
$$

By the comparison principle for parabolic equations, for any $0 \leq x \leq 1$ and $t>0, Z(x, t) \geq$ $u(x, t)$. As stated before, (2.11) has a positive steady state solution if and only if $d_{2}>\frac{q^{2}}{4 r}$ and $L>L^{*}\left(d_{2}\right)$. Clearly, this will not be satisfied for any $d_{2} \notin(\underline{d}, \bar{d})$. So $(2.11)$ has no positive steady state, and we have $Z(x, t) \rightarrow 0$ as $t \rightarrow \infty$. Hence, $v(x, t) \rightarrow 0$ as $t \rightarrow \infty$. By the first equation in (2.8) and the classical regularity theory of parabolic equations, we have $u(x, t) \rightarrow \tilde{u}$ as $t \rightarrow \infty$.

(b) The argument is exactly the same as that in the proof of part (a).

(c) If $d_{1}$ and $d_{2}$ are not in $(\underline{d}, \bar{d})$, then both the semi-trivial steady states $(\tilde{u}, 0)$ and $(\tilde{v}, 0)$ do not exist. Then a similar argument to that in the proof of part (a) implies that $u(x, t) \rightarrow 0$ and $v(x, t) \rightarrow 0$ as $t \rightarrow \infty$. This completes the proof.

Theorem 2.2 tells us that a species with its dispersal rate within the interval $(\underline{d}, \bar{d})$ will drive its competitor with its dispersal rate outside this interval to extinction. In fact, this is not surprising since, by the proof of Theorem 2.2, there is only one semi-trival steady state in that situation. The more interesting case is when the dispersal rates of both species lie within the interval $(\underline{d}, \bar{d})$, and then both semi-trivial steady states $(\tilde{u}, 0)$ and $(\tilde{v}, 0)$ exist. To this end, we need some necessary preparations.

We begin by considering the eigenvalue problem of the steady state equation of (2.1). We have

$$
\left\{\begin{array}{l}
d \varphi_{x x}-q \varphi_{x}+r \varphi+\lambda \varphi=0, \quad 0<x<L, \\
d \varphi_{x}(0)-q \varphi(0)=0, \quad \varphi(L)=0 .
\end{array}\right.
$$

It is well known that the existence and uniqueness of a positive steady state for (2.1) are determined by the sign of the principal (the smallest) eigenvalue $\lambda_{1}=\lambda_{1}(d)$ of $(2.12)$ (see, 
e.g. [8]). More precisely, if $\lambda_{1}=\lambda_{1}(d)<0$, then (2.12) has a unique positive steady state $u^{*}$ which is globally asymptotically stable. If $\lambda_{1}=\lambda_{1}(d)>0$, then (2.12) has no positive steady state, and the trivial solution 0 is globally asymptotically stable. By the argument of Theorem 2.1 (also see [4]), and the proof of Theorem 2.2, we have

$$
\lambda_{1}(d) \begin{cases}<0 & \text { if } d \in(\underline{d}, \bar{d}), \\ =0 & \text { if } d=\underline{d} \text { or } d=\bar{d}, \\ >0 & \text { if } d<\underline{d} \text { or } d>\bar{d},\end{cases}
$$

and then we have the following conclusion.

Theorem 2.3 Assume that $L>L_{0}$, where $L_{0}=L^{*}\left(d_{0}\right)=\min \left\{L^{*}(d): d>\frac{q^{2}}{4 r}\right\}$. Then the following statements are equivalent:

(1) the single species permits persistence for any non-negative initial value $u_{0}(x) \not \equiv 0$ in (1.1),

(2) the steady state equation of (1.1) has a unique positive steady state $u^{*}$ which is globally asymptotically stable,

(3) the dispersal rate $d$ lies within the open interval $(\underline{d}, \bar{d})$,

(4) the principal eigenvalue $\lambda_{1}(d)$ of $(2.12)$ is positive.

Now we go back to study competition system (2.8) and its steady state equation (2.9). Suppose that the semi-trivial steady state solution $(\tilde{u}, 0)$ exists. Then its stability is determined by the principal eigenvalue of the following eigenvalue problem (see, e.g. [8]):

$$
\left\{\begin{array}{l}
d_{2} \varphi_{x x}-q \varphi_{x}+(r-\tilde{u}) \varphi+\mu \varphi=0, \quad 0<x<L \\
d \varphi_{x}(0)-q \varphi(0)=0, \quad \varphi(L)=0 .
\end{array}\right.
$$

Let $\mu_{1}\left(d_{2}, \tilde{u}\right)$ denote the principal eigenvalue of (2.14). Then it is well known that $(\tilde{u}, 0)$ is linearly stable if $\mu_{1}\left(d_{2}, \tilde{u}\right)>0$, and linearly unstable if $\mu_{1}\left(d_{2}, \tilde{u}\right)<0$ [8].

Similarly, the stability of the semi-trivial steady state solution $(0, \tilde{v})$ (if it exists) is determined by the principal eigenvalue, denoted by $\mu_{1}\left(d_{1}, \tilde{v}\right)$, of the following eigenvalue problem:

$$
\left\{\begin{array}{l}
d_{1} \varphi_{x x}-q \varphi_{x}+(r-\tilde{v}) \varphi+\mu \varphi=0, \quad 0<x<L \\
d \varphi_{x}(0)-q \varphi(0)=0, \quad \varphi(L)=0 .
\end{array}\right.
$$

We now state our stability result of semi-trivial solutions for $d_{1}, d_{2} \in(\underline{d}, \bar{d})$ with $d_{1}<d_{2}$.

Theorem 2.4 Suppose that $L>L_{0} \equiv \min \left\{L^{*}(d): d>\frac{q^{2}}{4 r}\right\}$, $\underline{d}$ and $\bar{d}$ are defined as in Theorem 2.2 , and $d_{1}, d_{2} \in(\underline{d}, \bar{d})$ with $d_{1}<d_{2}$. Then the two semi-trivial steady state solutions $(\tilde{u}, 0)$ and $(0, \tilde{v})$ exist. For any fixed $d_{2} \in(\underline{d}, \bar{d})$, there exists a constant $\delta>0$, such that for any $d_{1} \in(\underline{d}, \underline{d}+\delta) \subset\left(\underline{d}, d_{2}\right)$, the solutions $(\tilde{u}, 0)$ are linearly unstable.

Similarly, we have the following. 
Theorem 2.5 Suppose that $L>L_{0} \equiv \min \left\{L^{*}(d): d>\frac{q^{2}}{4 r}\right\}, \underline{d}$ and $\bar{d}$ are defined as in Theorem 2.2 , and $d_{1}, d_{2} \in(\underline{d}, \bar{d})$ with $d_{1}<d_{2}$. Then the two semi-trivial steady state solutions $(\tilde{u}, 0)$ and $(0, \tilde{v})$ exist. For any fixed $d_{1} \in(\underline{d}, \bar{d})$, there exists a constant $\delta>0$, such that for any $d_{2} \in(\bar{d}-\delta, \bar{d}) \subset\left(d_{1}, \bar{d}\right)$, the solutions $(0, \tilde{v})$ are linearly unstable.

Before giving the proofs of Theorem 2.4 and Theorem 2.5, we provide the following lemma.

Lemma 2.6 Suppose that $L>L_{0} \equiv \min \left\{L^{*}(d): d>\frac{q^{2}}{4 r}\right\}, \underline{d}$ and $\bar{d}$ are defined as in Theorem 2.2, and $d \in(\underline{d}, \bar{d})$. Then the problem (2.1) has a unique positive solution $u^{*}$. Moreover, $\int_{0}^{L} u^{* 2} \rightarrow 0$ as $d \rightarrow \underline{d}+0$ or $d \rightarrow \bar{d}-0$.

Proof The existence and uniqueness of a positive solution $u^{*}$ follow from Theorem 2.3. We now prove $\int_{0}^{L} u^{* 2} \rightarrow 0$ as $d \rightarrow \underline{d}+0$. For the case $d \rightarrow \bar{d}-0$, the argument is similar. For the contrary, there exist a positive constant $c$ and a dispersal sequence $d^{(k)} \in(\underline{d}, \bar{d})$, $d^{(k)} \rightarrow \underline{d}+0$, such that the corresponding positive steady state $u_{k}^{*}$ satisfies $\int_{0}^{L} u_{k}^{* 2} \geq c$. We have

$$
\left\{\begin{array}{l}
d^{(k)}\left(u_{k}^{*}\right)_{x x}-q\left(u_{k}^{*}\right)_{x}+u_{k}^{*}\left(r-u_{k}^{*}\right)=0, \quad 0<x<L, \\
d^{(k)}\left(u_{k}^{*}\right)_{x}(0)-q u_{k}^{*}(0)=0, \quad u_{k}^{*}(L)=0 .
\end{array}\right.
$$

It follows from the maximal principle that $u_{k}^{*}<r$. Then the standard regularity argument and Sobolev's embedding theorem imply that, subject to a subsequence if necessary, $u_{k}^{*}$ is weakly in $H^{1}([0, L])$ and strongly in $L^{2}([0, L])$ to some non-negative function $u^{*}$, and then $\int_{0}^{L} u^{* 2} \geq c$. By Schauder's regularity theory, letting $k \rightarrow \infty$ in equation (2.16), we have

$$
\left\{\begin{array}{l}
\underline{d} u_{x x}^{*}-q u_{x}^{*}+u^{*}\left(r-u^{*}\right)=0, \quad 0<x<L, \\
\underline{d} u_{x}^{*}(0)-q u^{*}(0)=0, \quad u^{*}(L)=0 .
\end{array}\right.
$$

By the maximal principle and the inequality $\int_{0}^{L} u^{* 2} \geq c, u^{*}>0$ in $[0, L)$, that is, problem (1.1) has a positive steady state solution for $d=\underline{d}$, a contradiction by Theorem 2.3. The proof is completed.

Proof of Theorem 2.4 For $d_{1}, d_{2} \in(\underline{d}, \bar{d})$, the existence of the semi-trivial solutions $(\tilde{u}, 0)$ and $(0, \tilde{v})$ follows directly from Theorem 2.3 . By the variational expression of the principal eigenvalue (see [8]), and the principal eigenvalue of the eigenvalue problem (2.14), we have

$$
\begin{aligned}
\mu_{1}\left(d_{2}, \tilde{u}\right) & =\inf _{\varphi \in S}\left\{\int_{0}^{L}\left(d_{2} \varphi_{x}^{2}+q \varphi_{x} \varphi-r \varphi^{2}\right) d x+q \varphi^{2}(0)+\int_{0}^{L} u^{*} \varphi d x\right\} \\
& \leq \inf _{\varphi \in S}\left\{\int_{0}^{L}\left(d_{2} \varphi_{x}^{2}+q \varphi_{x} \varphi-r \varphi^{2}\right) d x+q \varphi^{2}(0)\right\}+\sup _{\varphi \in S} \int_{0}^{L} u^{*} \varphi d x,
\end{aligned}
$$

where $S=\left\{\varphi: \varphi \in H^{1}([0, L]), \varphi(0)=0, \int_{0}^{L} \varphi^{2}=1\right\}$.

Now by considering the variational expression of the principal eigenvalue $\lambda_{1}\left(d_{2}\right)$ of

$$
\left\{\begin{array}{l}
d_{2} \varphi_{x x}-q \varphi_{x}+r \varphi+\lambda \varphi=0, \quad 0<x<L, \\
d_{2} \varphi_{x}(0)-q \varphi(0)=0, \quad \varphi(L)=0,
\end{array}\right.
$$


we have

$$
\lambda_{1}\left(d_{2}\right)=\inf _{\varphi \in S}\left\{\int_{0}^{L}\left(d_{2} \varphi_{x}^{2}+q \varphi_{x} \varphi-r \varphi^{2}\right) d x+q \varphi^{2}(0)\right\} .
$$

For a fixed $d_{2} \in(\underline{d}, \bar{d})$, by Theorem $2.3, \lambda_{1}\left(d_{2}\right)$ is a negative constant. We take a fixed positive constant $a<\left|\lambda_{1}\left(d_{2}\right)\right|$. For any $\varphi \in S$.

$$
\int_{0}^{L} u^{*} \varphi d x \leq\left(\int_{0}^{L} u^{* 2} d x\right)^{1 / 2}\left(\int_{0}^{L} \varphi^{2} d x\right)^{1 / 2}=\left(\int_{0}^{L} u^{* 2} d x\right)^{1 / 2} .
$$

Then, by Lemma 2.6 , there exists a positive constant $\delta$, such that if $d_{1} \in(\underline{d}, \underline{d}+\delta)$, $\left(\int_{0}^{L} u^{* 2} d x\right)^{1 / 2}<a$. Combined with equations (2.19) and (2.20), we have

$$
\mu_{1}\left(d_{2}, \tilde{u}\right) \leq \lambda_{1}\left(d_{2}\right)+a<0
$$

This completes the proof of Theorem 2.4.

The proof of Theorem 2.5 is similar to that of Theorem 2.4 and thus omitted.

Remark 2.2 Note that Theorem 2.2 deals with the competitive exclusion situation, namely a species with dispersal rate in the interval $(\underline{d}, \bar{d})$ always drives its competitor with dispersal rate outside the interval to extinction. But Theorem 2.4 and Theorem 2.5 just give some competitive invasion results. If the dispersal rates $d_{1}$ and $d_{2}$ both lie within the interval $(\underline{d}, \bar{d})$, then any one of the two competitor species can evolve separately (without its competitor). Theorem 2.4 and Theorem 2.5 imply that a species with some intermediate dispersal rate in $(\underline{d}, \bar{d})$ can always invade its competitor with dispersal rate in $(\underline{d}, \bar{d})$ but close to one of the end points, regardless of the non-negative initial values $u(0, x), v(0, x) \not \equiv 0$. In this situation, competitive exclusion as well as coexistence may occur. For example, when $d_{1}, d_{2} \in(\underline{d}, \bar{d})$ and $d_{1}$ is close to $\underline{d}$ and $d_{2}$ close to $\bar{d}$, it seems that coexistence may happen, at least in some cases. The exact dynamical behavior in this situation seems subtle, and is likely related to a long time standing conjecture raised by Lou and Lutscher [5], which was also a main motivation for this paper.

\section{Concluding remarks}

This paper explores the optimal strategy of diffusion of a single-species model and a twospecies competition model in a 1-dimensional flowing homogeneous habitat with hostile downstream boundary conditions.

For the single-species model, we show that there exists an optimal diffusion rate $d_{0}$, in the sense that a species adopting the strategy of diffusion rate $d_{0}$ can be persistent with the smallest length of habitat.

For the two-species competition mode, given a fixed length of the habitat, there is a living interval $(\underline{d}, \bar{d})$ containing $d_{0}$ as an interior point, such that a species adopting the diffusion strategy and dispersal rates in this interval will replace its competitor with its dispersal rate outside the interval (Theorem 2.2). If the dispersal rates $d_{1}$ and $d_{2}$ both lie within the interval $(\underline{d}, \bar{d})$, then a species with some intermediate dispersal rate can always invade its competitor with its dispersal rate near the end points, regardless of the nonnegative initial values $u(0, x), v(0, x) \not \equiv 0$. These are remarkably different phenomena from those with downstream boundary conditions of type (I) and (II); see, for example, [5, 6]. 
Our work in this paper is mainly motivated by an open problem raised by Lou and Lutscher [5]. Lou and Lutscher [5] conjectured that there may exist an optimal intermediate diffusion rate $\tilde{d}$ so that a species adopting $\tilde{d}$ will replace its competitor with a different diffusion rate. Obviously, if the optimal rate $\tilde{d}$ does exist, then it must be in our living inter$\operatorname{val}(\underline{d}, \bar{d})$. Some further work is necessary to solve Lou and Lutscher's original conjecture.

\section{Acknowledgements}

We thank Prof. Yuan Lou and Dr. Peng Zhou for their helpful discussion and communication. The authors are grateful to the two anonymous reviewers for their excellent suggestions, which have greatly improved the exposition of the paper. This work is supported by the Natural Science Foundation of Shanghai, China (No. 13ZR1430100) and Shanghai Peak Subject Funding.

\section{Competing interests}

The authors declare that they have no competing interests.

Authors' contributions

All the authors read and approved the final manuscript.

\section{Publisher's Note}

Springer Nature remains neutral with regard to jurisdictional claims in published maps and institutional affiliations.

Received: 19 May 2017 Accepted: 23 August 2017 Published online: 02 September 2017

\section{References}

1. Anholt, BR: Density dependence resolves the stream drift paradox. Ecology 76, 2235-2239 (1995)

2. Hershey, AE, Pastor, J, Peterson, BJ, Kling, GW: Stable isotopes resolve the drift paradox for Baetis mayflies in an Arctic river. Ecology 74, 2315-2325 (1993)

3. Waters, TF: The drift of stream insects. Annu. Rev. Entomol. 17, 253-272 (1972)

4. Speirs, DC, Gurney, WSC: Population persistence in rivers and estuaries. Ecology 82, 1219-1237 (2011)

5. Lou, Y, Lutscher, F: Evolution of dispersal in open advective environments. J. Math. Biol. 69, 1319-1342 (2014)

6. Lam, K-Y, Lou, Y, Lutscher, F: Evolution of dispersal in closed advective environments. J. Biol. Dyn. (2014). doi:10.1080/17513758.2014.969336

7. Lou, Y, Zhou, P: Evolution of dispersal advective homogeneous environments: the effect of boundary conditions. J. Differ. Equ. 259(1), 28-29 (2015)

8. Cantrell, RS, Cosner, C: Spatial Ecology Via Reaction-Diffusion Equations. Series in Mathematical and Computational Biology. Wiley, Chichester (2003)

\section{Submit your manuscript to a SpringerOpen ${ }^{\odot}$ journal and benefit from:}

- Convenient online submission

- Rigorous peer review

- Open access: articles freely available online

- High visibility within the field

- Retaining the copyright to your article 